\title{
Ontology Based Construction of Bayesian Network
}

\section{Sonika Malik}

\begin{abstract}
In ontology engineering tasks such as modeling of domain, ontology reasoning and mapping of concepts between ontology, dealing with uncertainty is vital. Bayesian networks are used to determine the likelihood of occurrences affected by different factors. In this paper the concept of ontology to Bayesian network conversion requires the following tasks: i) determining the relevant nodes, ii) relationships between the factors recognized (connections), iii) for each node with in the Bayesiar network calculates the CPT's. Bayesian networks can capture and enhance the mixed way interdependence between techniques for ontology mapping. We outline the basic idea behind our approach and show some experiments on upper ontology. Then will focus on probabilistic ontology and their relationship with Bayesian Networks. At last it shows how the uncertainty can be handled by Bayesian network notion.
\end{abstract}

Index Terms: Bayesian Network, Directed Acyclic Graph Ontology, Semantic Web.

\section{I.INTRODUCTION}

Uncertainty is the concern of every aspect of semantic web ontologies. The uncertainty can make a significant contribution to the complexity of the chance. With reference to the Neches 'ontology definition, it defines the fundamental terms and relationships that comprise the vocabulary of a subject as well as the rules for the combination of terms and relations that define the vocabulary extensions. In order to integrate real world ontologies play a part. As a consequence, semantic heterogeneity is noted at ontological level, which is one of the primary barriers to the semantic web. In such a situation, ontology mapping is the key element of methods that attempt to address this issue. This includes finding mappings from distinct ontologies between entities (Ding, 2004). Most current ontology mapping systems, as can be seen, combine different techniques to accomplish high efficiency. Our method is based on the well-known method of Bayesian networks (BNs) that can detect interconnections between and within random variables. Bayesian networks, however, are not a standard depiction and the devices do not understand it effectively. Therefore, we try to propose a way to formally represent a Bayesian network as ontology with a standard OWL representation.

Manuscript revised on December 25, 2019 and published on January 10, 2019

Sonika Malik, Department of Information Technology, Maharaja Surajmal Institute of technology, Delhi, India. sonika.malik@gmail.com
The W3C Web Ontology Language (OWL) is a Semantic Web language that represents understanding, communities and relationships (Web Ontology Working Group, 2004).

We propose a method for the ontology based generation of Bayesian network:

By using ontological concepts to create the Bayesian network nodes

To connect the Bayesian network node through ontological relations, and

To develop CPT's for each node using ontology understanding (Neapolitan, 2003).

The paper is structured as follows. Section II briefly explains the notion of Bayesian networks, section III introduces the methodology to construct conditional probability tables, section IV explains the key rules to convert ontology to Bayesian Network, section $\mathrm{V}$ gives a clear view of work done so far and finally section VI concludes the research done on the topic.

\section{II.RELATED WORK}

We give a brief overview of approaches to ontology based Bayesian Networks that can be used to learn about Bayesian Networks from their outcomes.

Lam and Bacchus (Lam, 1994) defined an approach based on a minimal description length (MDL) for Bayesian Networks in order to learn relevant data. The approach requires no prior distribution assumptions and permits a compromise of precision with complexity in the model learned. Larrafiaga et al. (Larrafiaga, 1996) suggested a Bayesian network learning method using case databases to look for the best order of the function variables as input, and standard algorithms. Friedman and Koller (Friedman, 2003) introduced a Bayesian approach to Bayesian network technology exploration. On the basis of the data set, the method describes subsequent distributions through Bayesian network systems and assesses the future likelihood of important distribution structural features. Hruschka et al. (Ebecken, 2007) suggested an algorithm based on low computational complexity, which can be used to evaluate effective variables in a Bayesian learning network context. Helsper et al (Helsper, 2002) create Bayesian networks in four main phases: firstly, the Bayesian network's visual architecture emerges from ontological categories and functions. Classes are then transformed with an exhaustive, mutually excluded, discrete value condition of the groups in numerical variables. The third stage consists of converting properties into arcs between variables. Eventually, a handson integration process means that the Bayesian network 


\section{International Journal of Research in Advent Technology, Vol.7, No.12, December 2019 E-ISSN: 2321-9637 \\ Available online at www.ijrat.org}

eliminates redundant arcs and state spaces. The approach does not help visual form quantification (i.e. tables with the conditional probability). Ding et al (Ding, 2004) suggest probabilistic OWL mark-ups which can be applied in the OWL ontology to individual's classes and properties. The authors described a sequence of rules to translate the OWL ontology into DAG. CPT's for the each network node are built on the logical properties of its parent node. The approach presented in this article contrasts with the existing approaches:

- The graphic Bayesian Network Structure needs no special extensions

- It is a common technique and a model for constructing Bayesian networks on the basis of current OWL ontology.

- The necessary ontological extensions have no influence on existing classes and ontological individuals.

\section{BAYESIAN NETWORK}

A Bayesian network of $\mathrm{n}$ parameters comprises of a direct acyclic graph, which has n nodes and set of arcs as a whole. $\mathrm{X}_{\mathrm{i}}$ nodes equate to variables in a DAG, and direct arcs between two nodes indicate a direct causal relationship between one node and the other. The uncertainty of this relationship is localized by $\mathrm{CPT} \mathrm{P}\left(\mathrm{X}_{\mathrm{i}} \mid \Pi_{\mathrm{i}}\right)$ for each node $\mathrm{Xi}$ where $\Pi_{i}$ is the parent set of $\mathrm{Xi}$. At least in theory, BN accepts some assumption in the mutual probability distribution. Although the probabilistic inference with the general structure of DAG has been shown to be NP-hard (Cooper, 1990), BN inferential algorithms including belief propagation (Pearl, 1986) and junction tree (Lauritzen, 1988) were developed for BN's causal structures for successful calculation.

It is helpful to add some simple mathematical notation for parameters and distributions of probabilities. The parameters are shown with upper case letters $(\mathrm{A}, \mathrm{B}, \mathrm{C})$ and lower case letters $(\mathrm{a}, \mathrm{b}, \mathrm{c})$ for their meanings. If $\mathrm{A}=\mathrm{a}$, they say $\mathrm{A}$ was instantiated. The bold upper-cases letter $(\mathrm{X})$ is a number of variables and the bold lower-cases letter $(\mathrm{x})$ is a specific set of variables. If, for example, $\mathrm{X}$ indicates $\mathrm{A}, \mathrm{B}, \mathrm{C}$ then $\mathrm{x}$ is the instantiation $\mathrm{a}, \mathrm{b}, \mathrm{c} .|\mathrm{X}|$ is denoted as number of variables in $\mathrm{X} .|\mathrm{A}|$ is denoted as the number of possible states of $\mathrm{a}$. discrete variable $A$. The parent of $X$ in a graph is referred by $\mathrm{P}(\mathrm{X}) . \mathrm{P}(\mathrm{A})$ is used to denote the probability of $\mathrm{A}$.

For the joint probability denotation $\mathrm{P}(\mathrm{A}, \mathrm{B})$ and $\mathrm{P}(\mathrm{A} \mid \mathrm{B})$ is used and for conditional probability for the given variables A \& B. For e.g, if A is unambiguous, then $\mathrm{P}$ (A) may be equal to states $\{0.2,0.8\}$ i.e. $20 \%$ chance of truth and $80 \%$ chance of false. A joint probability means the likelihood of the existence of more than one parameter, as $\mathrm{A}$ and $\mathrm{B}$, referred to as $\mathrm{P}(\mathrm{A}, \mathrm{B})$.

An example of joint probability distribution for variables Raining and Windy is shown below in Table 1. For example, the probability of it being windy and not raining is 0.28 .
Table I: Joint Probability Distribution Example

\begin{tabular}{|l|l|l|}
\hline Raining & Wind=False & Wind=True \\
\hline True & 0.1 & 0.9 \\
\hline False & 0.72 & 0.28 \\
\hline
\end{tabular}

The conditional probability is the likelihood of a variable, given by another parameter, called $(\mathrm{A} \mid \mathrm{B})$. For example, the probability of Windy being True, given that Raining is true might equal $50 \%$.

$\mathrm{P}($ Windy $=$ True $\mid$ Raining $=$ True $)=50 \%$.

The whole theory of Bayesian network is based on Bayes theorem that permits us to define the conditional probability of evidence observing the cause based on the evidence.

$\mathrm{P}[$ Cause $\mid$ Evidence $]=\mathrm{P}$ [Evidence $\mid$ Cause $] \cdot \mathrm{P}$ [Cause $] \mathrm{P}$ [Evidence]

Each Bayesian network node is separate from its nondescendants, provided that the parents have been in the node. As Bayesian network is a function of probability, we can use total likelihood as the criterion of statistical knowledge. The highest probability estimation is a process that calculates values for model parameters. The parameter values are calculated so that the probability of the system as defined by the model is maximized.

The benefit of the Bayesian network is that it handles uncertainty in a tactful way as compare to other approaches.

\section{A. Ontology Based construction of Bayesian network}

For the development of Bayesian networks, the current ontological method includes four major phases 1) select the involved classes, individuals and properties, 2) Bayesian Network Structure creation 3) Conditional probability Table Creation 4) Inclusion of existing information

\section{Select the involved classes, Individuals and Properties:}

Every ontology class is well-defined, but a domain expert must select those classes, individuals and properties that are important to the problem considered and should be represented within the Bayesian networks, although they are semantically clear. Classes, individuals and properties are relevant in this context as they affect the state of the final output nodes of the Bayesian network (Fenz, 2012). The domain expert must ensure that no redundant edges in the Bayesian network are produced by selecting relevant classes, individuals and properties. E.g. if A is influenced by $\mathrm{B}$ and $\mathrm{B}$ by $\mathrm{C}$ only the edges of $\mathrm{B}$ to $\mathrm{A}, \mathrm{C}$ to $\mathrm{B}$ are permitted. The additional edge of $\mathrm{C}$ to $\mathrm{A}$ should not be permitted.

The domain expert has to select three different class/individual types, 1) Node Class/Individual which is 
directly related to problem domain 2) state space class/individual that defines the node class/individual state spaces 3) Weight Class/Individual define the node classes/individual vector weights.

To build a Bayesian network, selection of nodes and state space class is required.

- The number of potentials integrated into the Bayesian network is constrained by the choice of groups and individuals in the previous step. We use the category / classes previously defined to identify four various types of property for the building of Bayesian networks.

- Link Properties are those that connect the class/individual of the node selected in the previous step.

- State Value properties are those that provide the numerical values for discrete states defined by state space classes that are mutually exclusive.

- Weight Property: For the CPT calculation the weights are required for the appropriate child-parent combination.

In addition to the static knowledge model, ontology provides a dynamic knowledge base in order to integrate current knowledge as findings into the Bayesian network. The node and class properties are used for incorporating the findings in the Bayesian network.

\section{B. Bayesian Network Structure creation}

This step finally leads to the Bayesian network structure that is to say that a DAG with nodes and links. For each built-in node we use 1) State Space Classes / Individuals to define node state space and allocate numerical values for each state using the State Value Property selected, 2) Connect the node to its parent nodes by link Properties. The numerical values are required to calculate the CPTs of the Bayesian network nodes.

\section{CPT Construction}

The conditional probability table (CPT) is represented in order to display the conditional probabilities of one variable relative to others for a set of linear and mutually reliant random variables. Bayesian network inference involves the computation of the conditional probability for certain variables, with data on other variables (evidences). This is straightforward if all evidence available is for variables which are ancestral to variable(s) of interest.

We run the entire network to create the CPTs for every related node and pick those nodes with more than null parents. We use the weight classes / individuals and properties for each selected node to determine its parent nodes ' weights. For every child-parent node combination the tertiary pattern is used to describe the weight of the parent node. The CPT configuration of each node depends on i) its parent node's state space, (ii) its parent node's background weight and (iii) the distribution function, which specifies the use of the parent States to decide the node's state. When the parent node is limited to 2 states, the measurement complexity is minimized, i.e. there are two node states that have parent nodes, for example either "yes" or "no". Nodes without parents can have more than one or two states, for example "high", "medium" and "low". Every condition should be related to a numerical value lowest state. For computing probabilities we can use two laws either by law of total probability or chain rule of probability by taking a small example (Zhang, 2009).

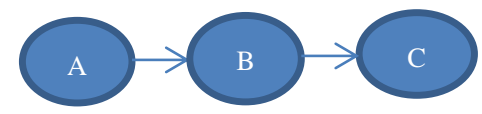

The prior probability "P(A)" be attached to a class "A" if it does not have any parent, conditional probability "P(A|B)" be attached to a class " $A$ " if it is a subclass of class " $\mathrm{B}$ " (so, $\mathrm{P}(\mathrm{A} \mid \mathrm{B})=0)$, and if necessary " $\mathrm{P}(\mathrm{A} \mid \mathrm{B})$ " are attached to a class "A" if it is disjoint with class " $\mathrm{B}$ " (so, $\mathrm{P}(\mathrm{A} \mid \mathrm{B})=0)$

\section{Law of Total Probability}

$$
\mathrm{P}(\mathrm{A})=\Sigma \mathrm{B} \mathrm{P}(\mathrm{A}, \mathrm{B})=\Sigma \mathrm{B} \mathrm{P}(\mathrm{A} \mid \mathrm{B}) \mathrm{P}(\mathrm{B})
$$

Where B is any random variable

\section{Chain Rule Of Probabilities}

We can also write

$$
\begin{gathered}
\mathrm{P}(\mathrm{A}, \mathrm{B}, \mathrm{C} \ldots \mathrm{Z})=\mathrm{P}(\mathrm{A} \mid \mathrm{B}, \mathrm{C} \ldots \mathrm{Z}) \mathrm{P}(\mathrm{B}, \mathrm{C} \ldots \mathrm{Z})(\text { by } \\
\text { definition of joint probability })
\end{gathered}
$$

Repeatedly applying this idea, we can write $\mathrm{P}(\mathrm{A}, \mathrm{B}, \mathrm{C} \ldots \mathrm{Z})=\mathrm{P}(\mathrm{A} \mid \mathrm{B}, \mathrm{C} \ldots \mathrm{Z}) \mathrm{P}(\mathrm{B} \mid \mathrm{C} \ldots \mathrm{Z}) \mathrm{P}(\mathrm{C} \mid \ldots$ $\mathrm{Z})$...P (Z)

A joint distribution in a structured form is defined by Bayesian network. This depicts dependency / independence through a directed graph, in which nodes are random variables, edges are direct dependency and dependent relations of independence as graph structure.

Marginal probability: It is an unconditional probability with the event occurred with the probability $(\mathrm{P}(\mathrm{A}))$. It is not influenced by any other event.

Joint probability: $\mathrm{P}$ (A and B). It is the probability of event $\mathrm{A}$ and $\mathrm{B}$. This is the likelihood that two or more things will intersect. It can be written as $\mathrm{P}(\mathrm{A} \cap \mathrm{B})$.

Conditional probability: $P(A \mid B)$ is the likelihood of the event $\mathrm{A}$, when event $\mathrm{B}$ is present. 
Table Ii: Marginal Probability

\begin{tabular}{|c|c|}
\hline $\mathrm{C} 1=0$ & $\mathrm{C} 1=1$ \\
\hline $1-\mathrm{p} 1$ & $\mathrm{p} 1$ \\
\hline
\end{tabular}

\begin{tabular}{|c|c|}
\hline $\mathrm{C} 2=0$ & $\mathrm{C} 2=1$ \\
\hline $1-\mathrm{p} 2$ & $1-\mathrm{p} 2$ \\
\hline
\end{tabular}

Table Iii: Conditional Probability

\begin{tabular}{|l|l|l|c|}
\hline & & $\mathrm{C} 0=0$ & $\mathrm{C} 0=1$ \\
\hline $\mathrm{C} 1=0$ & $\mathrm{C} 2=0$ & $1-\mathrm{p} 00$ & $\mathrm{p} 00$ \\
\hline $\mathrm{C} 1=0$ & $\mathrm{C} 2=1$ & $1-\mathrm{p} 01$ & $\mathrm{p} 01$ \\
\hline $\mathrm{C} 1=1$ & $\mathrm{C} 2=0$ & $1-\mathrm{p} 10$ & $\mathrm{p} 10$ \\
\hline$; / / \mathrm{C} 1=1$ & $\mathrm{C} 2=1$ & $1-\mathrm{p} 11$ & $\mathrm{p} 11$ \\
\hline
\end{tabular}

\section{USE CASE : SUPER ONTOLOGY}

This application case builds on the existing ontology. We illustrate how a Bayesian network of any ontology is innovative using the suggested approach and its implementation.

This paper describes the structure of the universe and defines the significance of the real world through the super ontology (Malik, 2015). This ontology is a matter for any entity that exists in this world. All things of this universe are eternal, but undergo numerous changes. During these changes there is no damage, after reuse we receive another form (http: //www. Umich.edu, http://www.jainlibrary.org) An entity undergoes changes to synthetic or natural forms and modes. Of example, a person undergoes multiple changes such as infancy, young and aged through the growth process. Such improvements are naturally occurring within human beings.

Some of the entities of example ontology are shown in Figure 1.

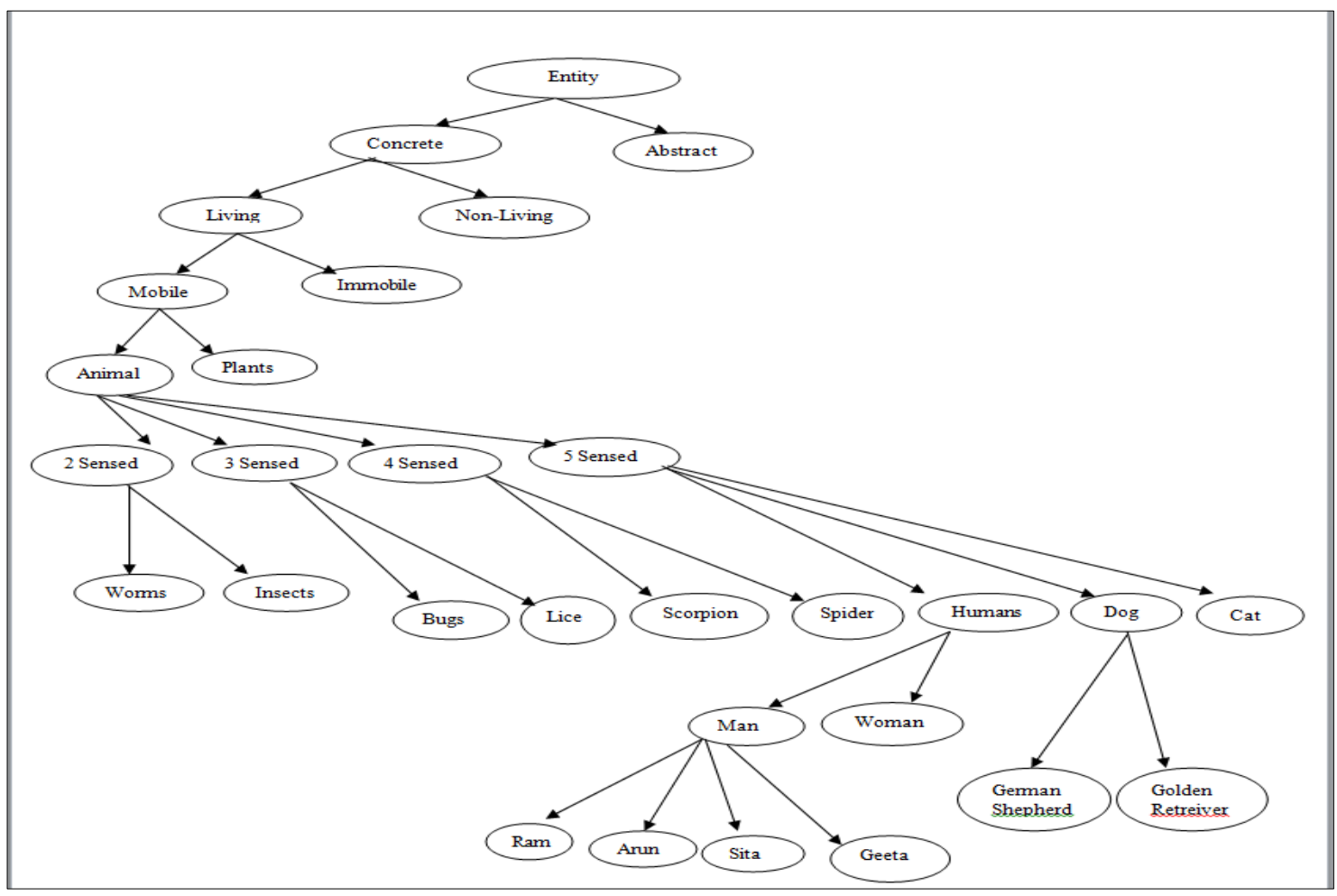

Fig. 1 : Super Ontology

This example seeks to determine the likelihood of the Bayesian network to show the Ontology Construction Process proposed. The user selects certain classes/individuals from the example ontology, based on the dependencies. For each node, the user selected a Boolean status space and specified the number of each status space. The next step is to construct the CPT construction of each node by using equation 1 .

$$
P_{B}\left(X_{1}, X_{2}, \ldots \ldots, X_{n}\right)=\prod_{i=1}^{n} P_{B}\left(X_{i} \mid \pi_{i}\right)=\prod_{i=1}^{n} \theta_{X_{i} \mid \pi_{i}}
$$

For the two events A \& B

$$
P(A \mid B)=\frac{P(A \cap B)}{P(B)}
$$




$$
\begin{gathered}
P(B \mid A)=\frac{P(A \cap B)}{P(A)} \\
P(A \mid B) P(B)=P(A \cap B)=P(B \mid A) P(A) \\
\mathrm{P}(\mathrm{A} \mid \mathrm{B})=\frac{P(B \mid A) P(A)}{P(B)}
\end{gathered}
$$

CPT for the node Animal with all its sub-classes like 2sensed, 3-sensed, 4-sensed and so on as given in the Table 4.

\section{TABLE IVA: CPT FOR ANIMAL WITH 2-SENSED}

\begin{tabular}{|l|l|l|}
\hline Animal & \multicolumn{2}{|l|}{ 2-sensed } \\
\hline & True & False \\
\hline True & 0.6 & 0.4 \\
\hline False & 0 & 1 \\
\hline
\end{tabular}

TABLE IVB: CPT FOR ANIMAL WITH 3-SENSED

\begin{tabular}{|c|c|c|}
\hline Animal & \multicolumn{2}{|c|}{ 3-sensed } \\
\hline & True & False \\
\hline True & 0.75 & 0.25 \\
\hline False & 0 & 1 \\
\hline
\end{tabular}

TABLE IVC: CPT FOR ANIMAL WITH 4-SENSED

\begin{tabular}{|l|l|l|}
\hline Animal & \multicolumn{2}{|l|}{ 4-sensed } \\
\hline & True & False \\
\hline True & 0.89 & 0.11 \\
\hline False & 0 & 1 \\
\hline
\end{tabular}

TABLE IVD: CPT FOR ANIMAL WITH 5-SENSED

\begin{tabular}{|c|c|c|}
\hline Animal & \multicolumn{2}{|c|}{ 5-sensed } \\
\hline & True & False \\
\hline True & 0.95 & 0.05 \\
\hline False & 0 & 1 \\
\hline
\end{tabular}

Now for the next level the CPT for each node is given in Table 5.

TABLE VA: CPT FOR 2-SENSED WITH WORMS

\begin{tabular}{|c|c|c|c|}
\hline Animal & $\begin{array}{c}2 \\
\text { sensed }\end{array}$ & \multicolumn{2}{|c|}{ Worms } \\
\hline & & True & False \\
\hline True & True & 0.5 & 0.5 \\
\hline True & False & 0.05 & 0.95 \\
\hline False & True & 0 & 1 \\
\hline False & False & 0 & 1 \\
\hline
\end{tabular}

TABLE VB: CPT FOR 2-SENSED WITH INSECTS

\begin{tabular}{|c|c|c|c|}
\hline Animal & $\begin{array}{c}2 \\
\text { sensed }\end{array}$ & \multicolumn{2}{|c|}{ Insects } \\
\hline & & True & False \\
\hline True & True & 0.5 & 0.5 \\
\hline True & False & 0.09 & 0.91 \\
\hline False & True & 0 & 1 \\
\hline False & False & 0 & 1 \\
\hline
\end{tabular}

There can be 2-sensed Animals like worms and insects and if the animals are not 2-sensed the $\mathrm{n}$ there is very less probablity that they are worms or insects. Similarly for 3sensed, 4-sensed and 5-sensed animals.

TABLE VC: CPT FOR 3-SENSED WITH BUGS

\begin{tabular}{|c|c|c|c|}
\hline Animal & $\begin{array}{c}\text { 3- } \\
\text { sensed }\end{array}$ & \multicolumn{2}{|c|}{ Bugs } \\
\hline & & True & False \\
\hline True & True & 0.5 & 0.5 \\
\hline True & False & 0.05 & 0.95 \\
\hline False & True & 0 & 1 \\
\hline False & False & 0 & 1 \\
\hline
\end{tabular}

\section{TABLE VD: CPT FOR 3-SENSED WITH LICE}

\begin{tabular}{|l|l|l|l|}
\hline Animal & \multicolumn{1}{|c|}{$\begin{array}{c}\text { 3- } \\
\text { sensed }\end{array}$} & \multicolumn{2}{|c|}{ Lice } \\
\hline & & True & False \\
\hline True & True & 0.55 & 0.45 \\
\hline True & False & 0.03 & 0.97 \\
\hline False & True & 0 & 1 \\
\hline False & False & 0 & 1 \\
\hline
\end{tabular}

TABLE VE: CPT FOR 4-SENSED WITH SCORPIO

\begin{tabular}{|c|c|c|c|}
\hline Animal & $\begin{array}{c}\text { 4- } \\
\text { sensed }\end{array}$ & \multicolumn{2}{|c|}{ Scorpio } \\
\hline & & True & False \\
\hline True & True & 0.85 & 0.15 \\
\hline True & False & 0.09 & 0.91 \\
\hline False & True & 0 & 1 \\
\hline False & False & 0 & 1 \\
\hline
\end{tabular}

TABLE VF: CPT FOR 4-SENSED WITH SPIDER

\begin{tabular}{|l|l|l|l|}
\hline Animal & \multicolumn{1}{|c|}{$\begin{array}{c}\text { 4- } \\
\text { sensed }\end{array}$} & \multicolumn{2}{|c|}{ Spider } \\
\hline & & True & False \\
\hline True & True & 0.5 & 0.5 \\
\hline True & False & 0.03 & 0.97 \\
\hline False & True & 0 & 1 \\
\hline False & False & 0 & 1 \\
\hline
\end{tabular}

TABLE VG: CPT FOR 5-SENSED WITH HUMAN

\begin{tabular}{|c|c|c|c|}
\hline Animal & $\begin{array}{c}\text { 4- } \\
\text { sensed }\end{array}$ & \multicolumn{2}{|c|}{ Human } \\
\hline & & True & False \\
\hline True & True & 0.99 & 0.01 \\
\hline True & False & 0.4 & 0.6 \\
\hline False & True & 0 & 1 \\
\hline False & False & 0 & 1 \\
\hline
\end{tabular}

TABLE VH: CPT FOR 5-SENSED WITH CAT

\begin{tabular}{|c|c|c|c|}
\hline Animal & $\begin{array}{c}5 \\
\text { sensed }\end{array}$ & \multicolumn{2}{|c|}{ Cat } \\
\hline & & True & False \\
\hline True & True & 0.55 & 0.45 \\
\hline True & False & 0.45 & 0.55 \\
\hline False & True & 0 & 1 \\
\hline False & False & 0 & 1 \\
\hline
\end{tabular}




$$
\text { E-ISSN: 2321-9637 }
$$

Available online at www.ijrat.org

Table VIA: CPT for 5-sensed with Dog

\begin{tabular}{|c|c|c|c|}
\hline Animal & $\begin{array}{c}5 \\
\text { sensed }\end{array}$ & \multicolumn{2}{|c|}{ Dog } \\
\hline & & True & False \\
\hline True & True & 0.7 & 0.3 \\
\hline True & False & 0.65 & 0.35 \\
\hline False & True & 0 & 1 \\
\hline False & False & 0 & 1 \\
\hline
\end{tabular}

After this we will come to the next level and CPT for the each node is given in Table 6 .

\section{TABLE VIB: CPT FOR 5-SENSED WITH HUMAN WITH MAN}

\begin{tabular}{|c|c|c|c|c|}
\hline Animal & 5 sense & Human & \multicolumn{2}{|c|}{ Man } \\
\hline & & & True & False \\
\hline True & True & True & 0.87 & 0.13 \\
\hline True & True & False & 0.3 & 0.7 \\
\hline True & False & True & 0.65 & 0.35 \\
\hline True & False & False & 0.45 & 0.55 \\
\hline False & True & True & 0 & 1 \\
\hline False & True & False & 0 & 1 \\
\hline False & False & True & 0 & 1 \\
\hline False & False & False & 0 & 1 \\
\hline
\end{tabular}

If 5 sensed animal is a human then it can either be Man or Woman.

TABLE VIC: CPT FOR 5-SENSED WITH HUMAN WITH WOMAN

\begin{tabular}{|l|l|l|l|l|}
\hline Animal & 5 sense & Human & \multicolumn{2}{|c|}{ Woman } \\
\hline & & & True & False \\
\hline True & True & True & 0.77 & 0.23 \\
\hline True & True & False & 0.41 & 0.59 \\
\hline True & False & True & 0.72 & 0.28 \\
\hline True & False & False & 0.25 & 0.75 \\
\hline False & True & True & 0 & 1 \\
\hline False & True & False & 0 & 1 \\
\hline False & False & True & 0 & 1 \\
\hline False & False & False & 0 & 1 \\
\hline
\end{tabular}

TABLE VID: CPT FOR 5-SENSED WITH HUMAN WITH MAN WITH RAM

\begin{tabular}{|c|c|c|c|c|c|}
\hline Animal & $\mathbf{5}$ sense & Human & Man & \multicolumn{2}{|c|}{ Ram } \\
\hline & & & & True & False \\
\hline True & True & True & True & 0.97 & 0.03 \\
\hline True & True & True & False & 0.55 & 0.45 \\
\hline True & True & False & True & 0.2 & 0.8 \\
\hline True & True & False & False & 0.25 & 0.75 \\
\hline True & False & True & True & 0.63 & 0.27 \\
\hline True & False & True & False & 0.6 & 0.4 \\
\hline True & False & False & True & 0.35 & 0.65 \\
\hline True & False & False & False & 0.3 & 0.7 \\
\hline False & True & True & True & 0 & 1 \\
\hline False & True & True & False & 0 & 1 \\
\hline False & True & False & True & 0 & 1 \\
\hline False & True & False & False & 0 & 1 \\
\hline False & False & True & True & 0 & 1 \\
\hline False & False & True & False & 0 & 1 \\
\hline False & False & False & True & 0 & 1 \\
\hline False & False & False & False & 0 & 1 \\
\hline
\end{tabular}

TABLE VIE: CPT FOR 5-SENSED WITH HUMAN WITH WOMAN WITH SITA

\begin{tabular}{|c|c|c|c|c|c|}
\hline Animal & $\mathbf{5}$ sense & Human & Woman & \multicolumn{2}{|c|}{ Sita } \\
\hline & & & & True & False \\
\hline True & True & True & True & 0.89 & 0.11 \\
\hline True & True & True & False & 0.4 & 0.6 \\
\hline True & True & False & True & 0.25 & 0.75 \\
\hline True & True & False & False & 0.42 & 0.58 \\
\hline True & False & True & True & 0.54 & 0.46 \\
\hline True & False & True & False & 0.61 & 0.39 \\
\hline rue & False & False & True & 0.4 & 0.6 \\
\hline True & False & False & False & 0.25 & 0.75 \\
\hline False & True & True & True & 0 & 1 \\
\hline False & True & True & False & 0 & 1 \\
\hline False & True & False & True & 0 & 1 \\
\hline False & True & False & False & 0 & 1 \\
\hline False & False & True & True & 0 & 1 \\
\hline False & False & True & False & 0 & 1 \\
\hline alse & False & False & True & 0 & 1 \\
\hline False & False & False & False & 0 & 1 \\
\hline
\end{tabular}

TABLE VIF: CPT FOR 5-SENSED WITH DOG WITH ANY SPECIES

\begin{tabular}{|c|c|c|c|c|}
\hline Animal & 5 sense & Dog & \multicolumn{2}{c|}{ Any Species } \\
\hline & & & True & False \\
\hline True & True & True & 0.8 & 0.2 \\
\hline True & True & False & 0.35 & 0.65 \\
\hline True & False & True & 0.54 & 0.46 \\
\hline True & False & False & 0.45 & 0.55 \\
\hline False & True & True & 0 & 1 \\
\hline False & True & False & 0 & 1 \\
\hline False & False & True & 0 & 1 \\
\hline False & False & False & 0 & 1 \\
\hline
\end{tabular}

TABLE VIG: CPT FOR 5-SENSED WITH CAT WITH ANY SPECIES

\begin{tabular}{|c|c|c|c|c|}
\hline Animal & 5 sense & Cat & \multicolumn{2}{|c|}{ Any Species } \\
\hline & & & True & Fals \\
\hline True & True & True & 0.85 & 0.15 \\
\hline True & True & False & 0.26 & 0.74 \\
\hline True & False & True & 0.65 & 0.35 \\
\hline True & False & False & 0.45 & 0.55 \\
\hline False & True & True & 0 & 1 \\
\hline False & True & False & 0 & 1 \\
\hline False & False & True & 0 & 1 \\
\hline False & False & False & 0 & 1 \\
\hline
\end{tabular}

After calculating all the probablities and CPT construction the final step is to construct the bayesian network for the existing ontology as in Figure 1. The translated Bayesian network from the super ontology is shown in Figure 2.

The provided use case developed a core Bayesian network of 120 nodes, 454 axioms and 119 links. It allows an expert in the field to build a Bayesian network based on existing ontology. The efficiency of the developed Bayesian network is evaluated for the use case. The usefulness of constructing bayesian network is i) It enabled them to build a bayesinan network effectively without any external help., ii) the information needed in ontology is centrally managed and transferred to the bayesian network. Finally the building steps for each node are: i) scanning the name of ontology 


\section{Available online at www.ijrat.org}

node and its type ii) state space include the allocation of numerical value (for example "true" or "false") iii) verifying

each node should be connected to the current node iv)

Linking of each node to its parent and its child.

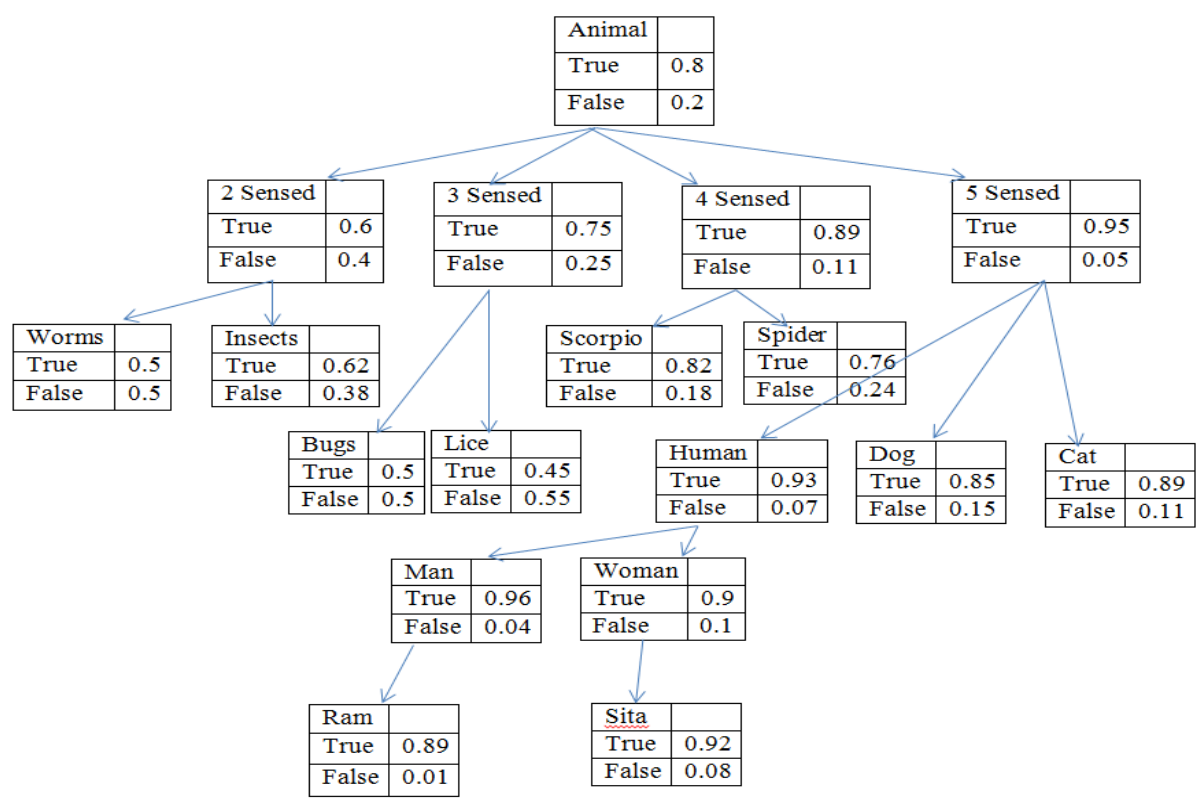

Fig. 2: Translated BN for Super Ontology

\section{CONClusion \& Future Work}

While creating the Bayesian Networks the following challenges are faced: i) What variables are required for any issue? ii) How to link these variables to each other? iii) What are the states of the determined variables? To overcome such problems, an ontology based approach for developing Bayesian networks is introduced and demonstrate its applicability for existing ontology. The proposed method enables the creation of Bayesian networks by giving the probablity at each node, which can handle uncertainty also.

The limitations of this method is: Ontology does not provide the functionsfor computing the CPT's, it must be explicitly modeled.

\section{REFERENCES}

[1] Z. Ding and Y. Peng, "A Probabilistic Extension to Ontology Language OWL," Proc. 37th Hawaii Int'l Conf. System Sciences (HICSS 04), IEEE CS Press, 2004.

[2] http://www.w3.org/2004/OWL

[3] R. Neapolitan. Learning Bayesian networks. Prentice Hall, 2003.

[4] W. Lam, F. Bacchus, "Learning Bayesian belief networks: an approach based on the mdl principle", Computational Intelligence, vol 10, 1994. 269-293.

[5] P. Larrafiaga, C. Kuijpers, R. Murga, Y. Yurramendi, "Learning Bayesian network structures by searching for the best ordering with genetic algorithms", IEEE Transactions on Systems, Man, and Cybernetics - Part A 26, 1996 487-493.

[6] N. Friedman, D. Koller, "Being Bayesian about network structure. a Bayesian approach to structure discovery in Bayesian networks", Machine Learning 50, 2003, 95-125.

[7] E.R.H. Jr., N.F. Ebecken, "Towards efficient variables ordering for Bayesian networks classifier", Data \& Knowledge Engineering, vol 63 (2), 2007 258-269.
[8] E.M. Helsper, L.C. van der Gaag, Building Bayesian networks through ontologies, in: F. van Harmelen (Ed.), ECAI 2002: Proceedings of the 15th European Conference on Artificial Intelligence, IOS Press, 2002, pp. 680-684.

[9] G. F. Cooper, "The computational complexity of probabilistic inference using Bayesian belief network," Artificial Intelligence, vol. 42, 1990, 393-405.

[10] J. Pearl, "Fusion, propagation and structuring in belief networks," Artificial Intelligence, vol. 29, 1986, 241-248.

[11] S. L. Lauritzen and D. J. Spiegelhalter, "Local computation with

a. Probabilities in graphic structures and their applications in expert systems," J. Royal Statistical Soc. Series B, vol. 50(2), 1988, 157224.

[12] S. Fenz. "An ontology-based approach for constructing Bayesian networks", Data \& Knowledge Engineering, volume 73, 2012, 73-88.

[13] S. Zhang, Y. Sun, Y. Peng, X. Wang, BayesOWL: A Prototypes System for Uncertainty in Semantic Web. In Proceedings of IC-AI, 678-684, 2009

[14] S. Malik, S. Jain, "Sup Ont:An upper ontology (Accepted for Publication)," IJWLTT, IGI Global, to be published.

[15] http://www.umich.edu/ umjains/jainismsimplified/chapter03.html.

[16] http://www.jainlbrary.org/JAB/11_JAB_2015_Manual_Finpdf.

\section{AUTHORS PROFILE}

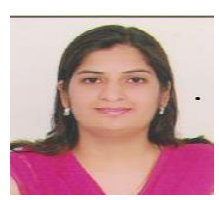

Sonika Malik has done B.Tech from Kurukshetra University, India in 2004 and did her Masters from MMU in 2010. She is doing her doctorate from National Institute of technology, Kurukshetra. She has served in the field of education from last 12 years and is currently working at Maharaja Surajmal Institute of Technology, Delhi. Her current research interests are in the area of Sematic Web, Knowledge representation and Ontology Design. 